\title{
The late-time radio spectrum of SN 1993J
}

\author{
M. A. Pérez-Torres ${ }^{1}$, A. Alberdi ${ }^{2}$, and J. M. Marcaide ${ }^{3}$ \\ 1 Istituto di Radioastronomia, via P. Gobetti 101, 40129 Bologna, Italy \\ e-mail: torres@ira.cnr.it \\ 2 Instituto de Astrofísica de Andalucía, CSIC, Apdo. Correos 3004, 18080 Granada, Spain \\ e-mail: antxon@iaa.es \\ 3 Departamento de Astronomía y Astrofísica, Universidad de Valencia, 46100 Burjassot, Spain \\ e-mail: J.M.Marcaide@uv.es
}

Received 16 May 2002 / Accepted 30 July 2002

\begin{abstract}
We present VLA radio continuum measurements of SN 1993J in M 81 at the frequencies of 0.32 ( $P$-band), 1.3 and 1.7 ( $L$-band), 4.9 ( $C$-band), 8.5 ( $X$-band), and 14.9 ( $U$-band) $\mathrm{GHz}$ carried out on December 17 and 21, 2000, about 2820 days after the supernova explosion. We find that a power-law spectrum, free-free absorbed by an homogeneous, or clumpy, distribution of ionized gas yields the best fit to the radio data. A combined homogeneous-clumpy model is not favored, but neither totally excluded. This result contrasts with the modeling of the early $(t \lesssim 230$ days) radio emission from SN 1993J, where a mixture of homogeneous and clumpy absorbers appeared to be necessary to adequately describe the behavior of the light curves. The radio spectrum of supernova SN 1993J between 0.32 and $14.9 \mathrm{GHz}$ is well characterized by $\alpha=-0.67 \pm 0.02$ $\left(S_{v} \propto v^{\alpha}\right)$, typical of an optically thin radio supernova. A fit to the radio spectra of SN 1993J from $\sim 70$ up to 2820 days shows that the observed spectral index of SN 1993J has been slowly evolving since $t \sim 1000$ days, with the observed spectral index changing from $\alpha \approx-1$ to $\alpha=-0.67$. This spectral evolution seems to suggest that, in addition to the radiative (synchrotron) losses, adiabatic cooling and ionization (Coulomb) losses at the lowest frequencies might be contributing significantly to the integrated electron spectrum.
\end{abstract}

Key words. techniques: interferometric - supernovae: individual: SN 1993J - ISM: supernova remnants radio continuum: stars - galaxies: individual: M 81

\section{Introduction}

The most widely accepted model for the radio emission from supernovae is the standard interaction model, in which the observed radio (synchrotron) emission is associated with the interaction region between the supernova ejecta and the presupernova wind of the progenitor star. The radio emission shows an early rise and a late decline due to a low-frequency absorption process. Chevalier (1982) considered a number of mechanisms that could account for the absorption: (i) freefree absorption by material in front of the forward shock front, (ii) free-free absorption by material within the emitting region, (iii) synchrotron self-absorption, and (iv) the Razin-Tsytovich effect. At the beginning of the 1980's, the available radio light curves could be well explained by synchrotron radio emission that was partially free-free absorbed by ionized thermal material in the circumstellar wind of the progenitor star (e.g. Weiler et al. 1986). The availability of more complete light curves for these supernovae (e.g. Weiler et al. 1989, 1990) provided further support for this mechanism. However, as new radio

Send offprint requests to: M. A. Pérez-Torres,

e-mail: torres@ira.cnr.it supernovae have been detected, the situation has become more complex. For example, the radio light curves of SN1986J showed a different shape in the rise to maximum, which was interpreted by Weiler et al. (1990) as evidence for internal free-free absorption. These authors suggested that a filamentary, clumpy-like structure of thermal absorbers could also be the source of the slow radio turn-on. Chevalier (1998) has recently argued that synchrotron self-absorption is likely to be the dominant absorption mechanism for some radio supernovae, in particular for SN1987A, and for type Ib and type Ic supernovae. Finally, observations of SN 1993J seem to indicate that more than one absorption mechanism plays a role (Fransson \& Björnsson 1998; Pérez-Torres et al. 2001; Mioduszewski et al. 2001).

SN 1993J is to date the radio supernova whose evolution has been monitored in greatest detail. The brightness and proximity of SN 1993J in M 81 offered an unprecedented occasion for VLBI studies, almost since the supernova explosion on 28 March 1993. For the first time, a shell-like structure in a young radio supernova was discovered (Marcaide et al. 1995a), in agreement with the circumstellar interaction model. Marcaide et al. (1995b) showed that the expansion of 
SN 1993J was self-similar, and produced the first movie of an expanding supernova. Later on, Marcaide et al. (1997) reported on the deceleration of the SN 1993J expansion, a result recently confirmed by Bartel et al. (2000). The modeling of the radio light curves of SN 1993J has also given deeper insight into the supernova phenomenon. It seems now clear that the progenitor of SN 1993J had a substantial mass-loss rate during the late stages of its evolution ( $\dot{M} \geq 10^{-5} M_{\odot} \mathrm{yr}^{-1}$, e.g. van Dyk et al. 1994). The power-law density profile of the circumstellar medium around SN 1993J seems to be different from the standard one (Lundqvist 1994; van Dyk et al. 1994; Marcaide et al. 1997; Immler et al. 2001; Mioduszewski et al. 2001), although Fransson \& Björnsson (1998) have succeeded in modeling the SN 1993J radio light curves with an $s=2$ density profile for the CSM.

Based on numerical modelings of the available radio data for SN 1993J, Fransson \& Björnsson (1998) and Pérez-Torres et al. (2001) made predictions about its late-time, low frequency $(<1.4 \mathrm{GHz})$ radio light behaviour. However, observational results of the low-frequency radio emission from SN 1993J were lacking. Thus, we carried out VLA observations between $\sim 322$ and $\sim 15000 \mathrm{MHz}$ aimed at checking the above modeling efforts, as well as a way to discern the main absorbing processes acting in SN 1993J.

\section{Observations and data reduction}

We observed SN 1993J using 25 antennas of the VLA in its most extended configuration (A, with baseline lengths from 0.68 up to $36.4 \mathrm{~km})$ at $L(18 \mathrm{~cm})$ and $P(90 \mathrm{~cm})$ bands from UT 13:55 to UT 15:25 on 17 December 2000 , and at $U(14.9 \mathrm{GHz})$, $X(8.5 \mathrm{GHz}), C(4.9 \mathrm{GHz}), L(1.3-1.7 \mathrm{GHz})$, and $P(0.32 \mathrm{GHz})$ bands from UT 08:40 to UT 11:40 on 21 December 2000. Each frequency band was split into two intermediate frequencies (IFs). Data processing was made using the Astronomical Image Processing System (AIPS). We first describe briefly the observations at the higher frequencies, and then emphasize and describe in detail those at lower frequencies, particularly those in the $P$-band, deemed to be a discriminator of the dominant absorption mechanism in SN 1993J. Table 1 summarizes our observations. The flux densities reported were obtained by combining the data from both intermediate frequencies (IFs) at each frequency band, except at $L$-band, for which the frequency separation was large enough that we could use each flux density value independently.

\subsection{High-frequency ( $U$-, $X$-, and $C$-band) observations}

We used a standard continuum mode at $U, X$, and $C$ bands, thus covering a bandwidth of $50 \mathrm{MHz}$ per IF and frequency band. At all three bands, we observed SN 1993J phase-referenced to $0917+624$. The source $0917+624$ also served as the phase reference for the system calibration. At $U$ - and $X$-band, 3C 286 was used as primary flux calibrator (assumed of constant flux density), while at $C$-band 3C 147 was used instead. At $U$-band, the integration times on SN 1993J and $0917+624$ were $\tau_{93 \mathrm{~J}}=$ $21 \mathrm{~min}$ and $\tau_{0917}=7 \mathrm{~min}$, while at $X$-band these were 11.5 and $6 \mathrm{~min}$, and at $C$-band 12 and $7 \mathrm{~min}$, respectively.

\subsection{Low-frequency ( $L$ - and P-band) observations}

Observations were done in spectral-line mode in order to detect the likely presence of radio frequency interference (RFI) in the data, and also to avoid bandwidth smearing. In addition, this observing mode allows a more careful editing. 3C 286 was used at both bands as primary flux calibrator, as well as bandpass calibrator.

L-band observations. Each of the two $12.5-\mathrm{MHz}$ IFs at $L$-band (centered at 1.34 and $1.67 \mathrm{MHz}$, respectively) were split into 8 spectral channels. Observations of SN 1993J $\left(\tau_{93 \mathrm{~J}}=11.5 \mathrm{~min}\right)$ were phase-referenced to $0917+624\left(\tau_{0917}=\right.$ $10.5 \mathrm{~min}) .0917+624$ was also used as secondary flux calibrator at $L$-band. Our final hybrid map at $L$-band (not shown here) was made at an effective observing frequency of $1.50 \mathrm{GHz}$. The peak of brightness of the map ( $97.4 \mathrm{mJy} \mathrm{beam}^{-1}$ ) corresponds to the nucleus of M 81, and the flux density of SN 1993J is $31.9 \mathrm{mJy}$. The rms of the image background is $0.3 \mathrm{mJy} / \mathrm{beam}$. The flux density values reported at $L$-band in Table 1 correspond to each IF, 1.34 and $1.67 \mathrm{GHz}$, respectively. Since the center frequencies in $L$-band are quite separated, they can be used as independent values for the purpose of fitting a spectral index (see below). We note that, though the distance between SN $1993 \mathrm{~J}$ and M $81\left(2^{\prime} 48^{\prime \prime}\right)$ is much larger than the dimensions of the restoring beam $(1.84 \times 0.99$ arcsec $)$, wide field mapping is necessary if one aims at accurate determinations of the flux densities of SN 1993J at low frequencies.

$P$-band observations. At $P$-band, each of the two 3.125-MHz IFs (centered at 322 and $327 \mathrm{MHz}$, respectively) was split into 32 spectral channels, which permitted a detailed inspection of the data, looking for the presence of RFI. Since $0917+624$ is a relatively weak source at $P$-band, unlike at higher frequencies, confusion of many strong sources in the field made it unsuitable as secondary calibrator. Therefore, the much stronger source J1206+6414 (3C 268.3) was used instead. The total integration times were $\tau_{93 \mathrm{~J}} \approx 78 \mathrm{~min}$, and $\tau_{3 \mathrm{C} 268.3} \approx 39 \mathrm{~min}$. We imaged $3 \mathrm{C} 268.3$ to obtain an accurate value of its flux density. We noticed that the coordinates used for $3 \mathrm{C} 268.3$ at the correlator $\left(\alpha=12^{\mathrm{h}} 06^{\mathrm{m}} 24^{\mathrm{s}} .711\right.$, $\delta=+64^{\circ} 14^{\prime} 40^{\prime}$ '939; J2000.0) were off from the actual ones by more than one arcmin. Indeed, the coordinates obtained using the AIPS task JMFIT were $\alpha=12^{\mathrm{h}} 06^{\mathrm{m}} 24.8978, \delta=$ $+64^{\circ} 13^{\prime} 36^{\prime \prime}$. 425 (J2000.0). Such position offset for 3C 268.3 translated into a similar one for SN 1993J and the whole sky around it. We corrected the phases of 3C 268.3 for this offset.

With the 3C 268.3 data corrected, we imaged SN 1993J at $P$-band, aimed to determine its flux density. Since the primary beam of the VLA at $P$-band is so large $\left(\approx 2^{\circ} 20^{\prime}\right)$, bright sources far from the supernova could greatly affect our image. Further, to obtain a good signal-to-noise ratio (SNR) in the image, one is forced to map even distant, strong sources. The situation is similar to that encountered at $L$-band, where self-calibration was mandatory to improve the SNR of the image, but useless unless M 81 was also included in the imaging process. At $P$-band, not only the M 81 nucleus is important, but also far away, strong sources such as M 82 need also to be taken into account to be able to apply self-calibration to the data. We used the AIPS task SETFC to create a list of fields with 


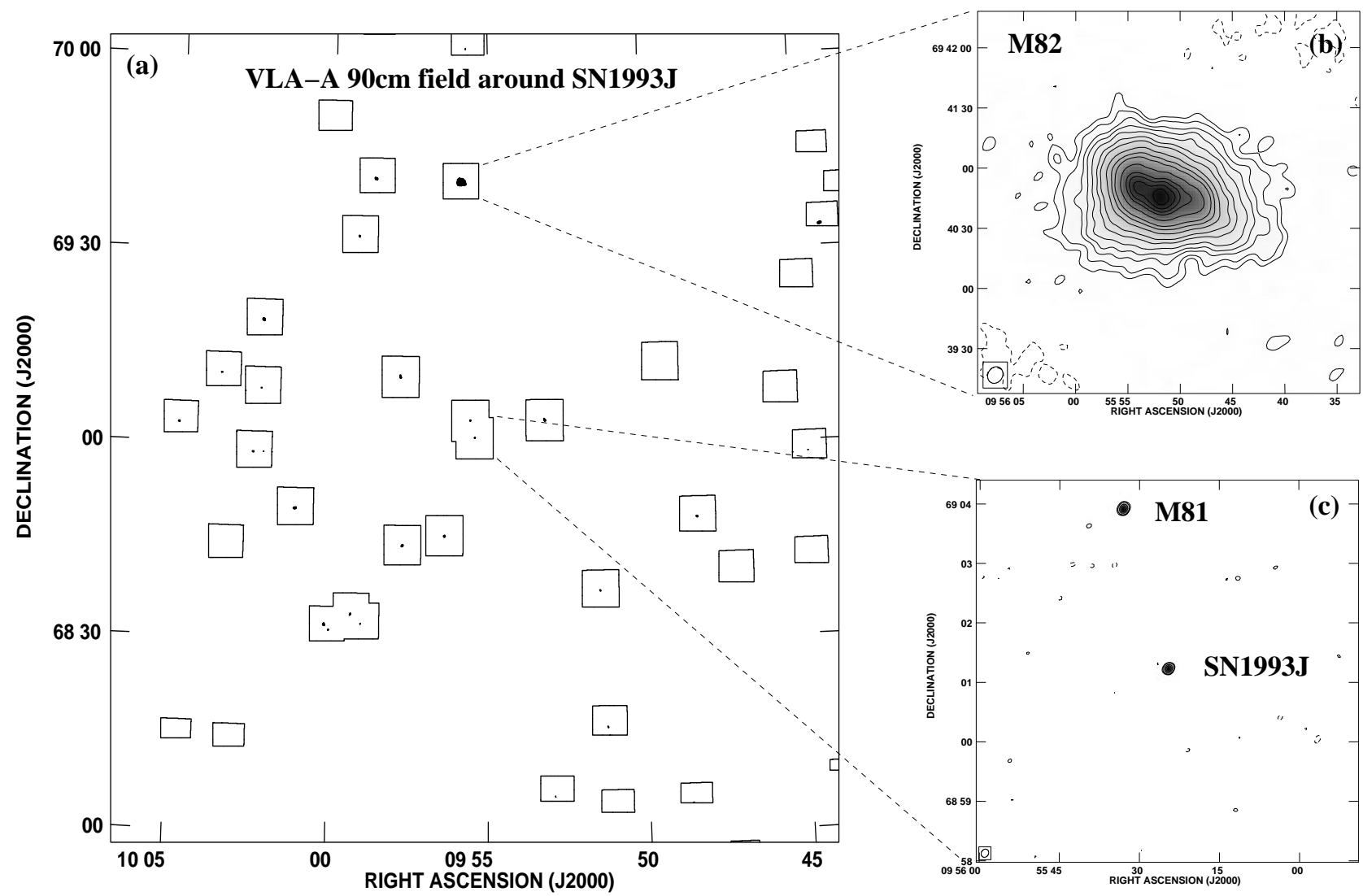

Fig. 1. a) Field of of view of the VLA in A configuration (VLA-A) around SN 1993J at $P$-band, on 17 and 21 December, 2000. The image shows only the inner $2 \times 2$ deg (rather than the $4 \times 4$ deg actually mapped), which includes 38 fields out of the 78 used in the hybrid mapping process. The image is centered on SN 1993J. Only contours above $20 \mathrm{mJy}$ beam $^{-1}$ have been drawn. b) Hybrid map of the galaxy M 82 . The contours are $(-2,2,3,5,7,10,15,20,30,40,50,70,90,100,110,120) \times 4.2 \mathrm{mJy}$ beam $^{-1}$, the off-source rms noise. The peak of brightness of the map $\left(\alpha=09^{\mathrm{h}} 55^{\mathrm{m}} 51^{\mathrm{s}} \mathrm{s} .14, \delta=+69^{\circ} 40^{\prime} 45^{\prime \prime} 20 ; \mathrm{J} 2000.0\right)$ is $537.8 \mathrm{mJy}$ beam ${ }^{-1}$. The flux density in the image above the $2-\mathrm{rms}$ level is $10.49 \mathrm{Jy}$. The dimensions of the restoring beam are $8.6 \times 7.2 \mathrm{arcsec}$, with the beam's major axis oriented along PA $=-30.2$ deg. c) Hybrid map of SN 1993J and the nucleus of its host galaxy M 81. The contours are $(-3,3,5,10,15,20,25,30) \times 3.3 \mathrm{mJy}^{\text {beam }}{ }^{-1}$, the off-source rms noise. The peak of brightness of the map corresponds to the supernova and is $71.1 \mathrm{mJy}$ beam $^{-1}$. The dimensions of the restoring beam are $8.6 \times 7.2$ arcsec, with the beam's major axis oriented along $\mathrm{PA}=-30.9 \mathrm{deg}$.

suspected sources in them, rather than making an enormous, computationally unmanageable image. It is worth noticing that the interferometric phases are initially so poorly calibrated that the reconstructed image will show very few sources, making very difficult the convergence of the hybrid mapping procedure. Therefore, we used the AIPS procedure FACES, which searches for sources in the NVSS catalog, to supply an initial model of the sky, and thus improve the global phase calibration. We then applied wide-field imaging on this self-calibrated dataset: since the array configuration looks different to sources in different parts of the (large) primary beam, we handled this by computing the uv-coverage differently for many small fields within the task IMAGR. Sources above a threshold of $33 \mathrm{mJy}$ (at a frequency of $1.4 \mathrm{GHz}$ ) were searched for as a priori candidates in the NVSS catalog, within a $2 \times 2$ deg region around SN 1993J. This resulted in 78 fields that were cleaned using wide-field mapping. Figure 1 shows the inner $1 \times 1 \mathrm{deg}$ of our final, composite image of the VLA-A field at $P$-band. For clarity, only contours above $20 \mathrm{mJy}$ beam $^{-1}$ have been drawn. Panel b shows the galaxy M 82, offset about 0'40 from SN 1993J. The latter is shown in panel $\mathrm{c}$ along with the nucleus of its host galaxy M 81. M 82 has a flux of $\sim 10.5 \mathrm{Jy}$, at an epoch when SN 1993J has a flux of a mere $71.1 \mathrm{mJy}$ (see Table 1 for details of the observations).

\section{The radio spectrum of SN 1993J}

We fitted the radio spectrum of SN 1993J obtained on 17 and 21 December 2000 using three models for its radio emission. These models are described by the equations

$S(v)=K_{1} v_{5}^{\alpha}$

$S(v)=K_{1} v_{5}^{\alpha} \mathrm{e}^{-\tau}$

$S(v)=K_{1} v_{5}^{\alpha}\left(1-\mathrm{e}^{-\tau^{\prime}}\right) / \tau^{\prime}$

where $\tau=K_{2} v_{5}^{-2.1}$, and $\tau^{\prime}=K_{3} v_{5}^{-2.1}$. These equations represent a simple power-law spectrum (model 1), a power-law spectrum free-free absorbed by a screen of homogeneously distributed ionized gas (model 2), and a power-law spectrum freefree absorbed by a "clumpy" medium (model 3). To facilitate comparisons with the modeling of van Dyk et al. (1994), we have used here their notation: $v_{5}$ is the frequency, in units of 
Table 1. VLA observing results.

\begin{tabular}{lcccccc}
\hline \hline Source Name & \multicolumn{7}{c}{ Flux density (mJy) } \\
\cline { 2 - 7 } & $14.94 \mathrm{GHz}$ & $8.46 \mathrm{GHz}$ & $4.86 \mathrm{GHz}$ & $1.67 \mathrm{GHz}$ & $1.34 \mathrm{GHz}$ & $0.324 \mathrm{GHz}$ \\
\hline SN 1993J & $6.7 \pm 0.1$ & $10.4 \pm 0.2$ & $14.7 \pm 0.4$ & $30.5 \pm 0.4$ & $33.7 \pm 0.4$ & $71.1 \pm 3.4$ \\
$3 \mathrm{C} \mathrm{286} 6^{(1)}$ & $3293 \pm 16$ & $4939 \pm 5$ & - & $13708 \pm 20$ & $15157 \pm 21$ & $25940 \pm 120$ \\
$0917+624^{(2)}$ & $1164 \pm 1$ & $1370 \pm 1$ & $1472 \pm 1$ & $1272 \pm 2$ & $1194 \pm 3$ & - \\
$3 \mathrm{C} 147^{(1)}$ & - & - & $7940 \pm 10$ & - & - & $25940 \pm 120$ \\
$3 \mathrm{C} 268.3^{(2)}$ & - & - & - & - & - & $10040 \pm 20$ \\
\hline
\end{tabular}

(1) Primary flux calibrator, ${ }^{(2)}$ phase and secondary flux calibrator. All flux density values reported here were obtained by imaging each source, and the quoted uncertainty for each flux value corresponds to the off-source rms noise in each image.

$5 \mathrm{GHz} ; \alpha$ is the observed spectral index; $K_{1}$ is the flux density at $5 \mathrm{GHz}$, in mJy; and $K_{2}$ and $K_{3}$ are the optical depths at $5 \mathrm{GHz}$ of the homogeneous and "clumpy" external absorbing media, respectively, at the epoch of our observations. Table 2 summarizes our results, and Fig. 2 shows the best fits to models 1 through 3. A simple power-law spectrum (model 1) yields a good fit to the data from $14.94 \mathrm{GHz}$ down to $1.34 \mathrm{GHz}$, but departs significantly from the actual flux density at frequencies below $400 \mathrm{MHz}$, and can be ruled out. The best fits are given by models 2 (homogeneous absorbing medium) and 3 (clumpy absorbing medium), which yield an observed spectral index $\alpha=-0.67 \pm 0.02$. The frequency at which the free-free optical depth becomes unity is very similar for both model 2 $\left(v_{\tau=1}=169 \mathrm{MHz}\right)$ and model $3\left(v_{\tau=1}=241 \mathrm{MHz}\right)$. Note, however, that the very-low radio frequency behavior of the spectrum is significantly different (see Fig. 2). While model 2 (solid line) predicts a sharp fall-off below frequencies of $\sim 200 \mathrm{MHz}$, model 3 (dashed line) predicts a much milder flux density decrease. Unfortunately, the absence of radio measurements below $322 \mathrm{MHz}$ does not allow us to rule out either of these models. Thus, from Table 2 we cannot exclude, or prefer, either of the two alternative models.

To model the early-time ( $t \lesssim 230$ days) radio light curves of SN 1993J, van Dyk et al. (1994) invoked a combined "homogeneous-clumpy" model of the form $S_{v} \propto v_{5}^{\alpha} \mathrm{e}^{-\tau}(1-$ $\left.\mathrm{e}^{-\tau^{\prime}}\right) / \tau^{\prime}$. For such model, we can also obtain acceptable solutions (e.g. $\alpha=-0.67 ; K_{1}=14.4 ; K_{2}=8 \times 10^{-4} ; K_{3}=10^{-5}$ ) at the expense of increasing $\chi_{\text {red }}^{2}$ by $\approx 50 \%$. If the fitting parameters are left unconstrained, this model yields an even better fit than models 2 and 3 , but at the unaffordable price of a negative - hence unphysical - value for $K_{2}$, or $K_{3}$. Clearly, the combined "homogeneous-clumpy" model cannot be preferred over models 2 or 3, but we also feel we cannot exclude it solely on the basis of a $50 \%$ increase of the reduced $\chi^{2}$.

For a power-law relativistic electron distribution, $N_{E}=$ $N_{0} E^{-\gamma}$, the spectral index of the electron distribution $\gamma$ is related to the observed spectral index: $\gamma=1-2 \alpha$. From our value of $\alpha$, it then follows that $\gamma=2.34 \pm 0.04$. This value of $\gamma$ agrees well with that predicted by Fransson \& Björnsson (1998) for SN 1993J at epochs $t \gtrsim 1000$ days. We should note that synchrotron self-absorption could play a role in the radio emission from SN 1993J (Chevalier 1998; Fransson \& Björnsson 1998; Pérez-Torres et al. 2001; Mioduszewski et al. 2001). In particular, the modeling of Fransson \& Björnsson (see their Fig. 12),

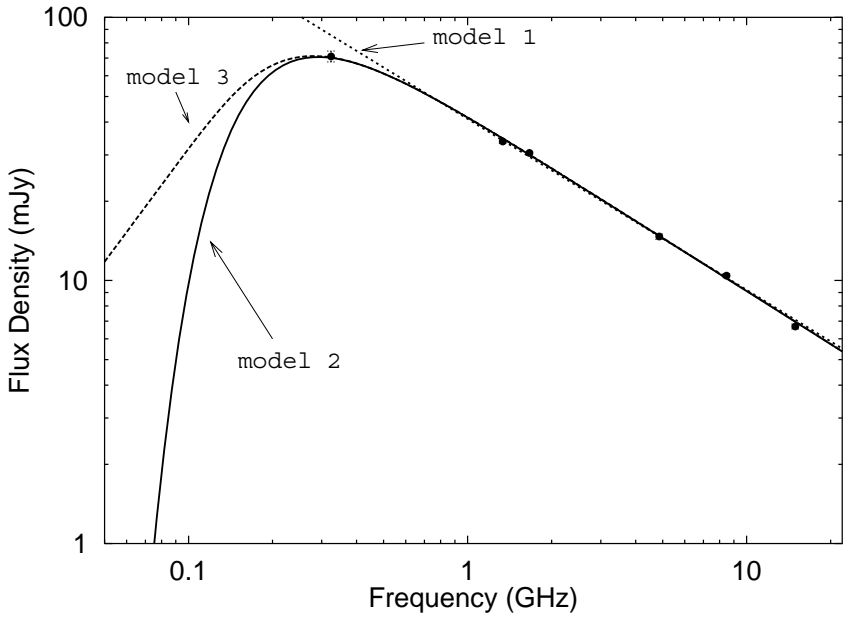

Fig. 2. Fits to the continuum radio spectrum of SN 1993J on 17 and 21 December 2000. As indicated in the figure, the dotted, solid, and dashed lines correspond to models 1,2 , and 3, respectively, as described in the text. Note that the size of each data point but the one at $0.324 \mathrm{GHz}$, is larger than its associated error bar.

predicted a flux density of $\sim 70 \mathrm{mJy}$ at $0.32 \mathrm{GHz}$ and for the approximate epoch of our observations, while the modeling of Pérez-Torres et al. predicted at that frequency a flux density larger than $100 \mathrm{mJy}$. Such an excess in flux of the latter modeling is likely related to the different spectral evolution obtained for the electron spectrum.

Figure 3 shows the evolution of the observed spectral index of SN 1993J from $\sim 70$ up to 2820 days after the explosion. For each epoch, we fitted the available radio continuum data (van Dyk, private communication) using model 2 as described above. While at early epochs the fits are not superb, they seem to be compatible with $\alpha \approx-1$. However, for epochs $\gtrsim 1000$ days, there seems to be a clear trend: the observed spectral index, $\alpha$, becomes progressively less steep. This spectral evolution could be explained assuming significant energy losses, mainly associated with synchrotron, as well as Coulomb and expansion losses. Fransson \& Björnsson (1998) proposed a model that reproduces reasonably well the observed spectral evolution of SN 1993J. In their model, a constant fraction of the shocked thermal electrons - characterized by a constant spectral index - are injected and accelerated. These electrons lose their energy mainly due to synchrotron losses, thus steepening the integrated electron spectrum. Moreover, these authors 
Table 2. Radio spectrum fits for the VLA observations of SN 1993J on 17 and 21 December 2000.

\begin{tabular}{lccccccc}
\hline \hline Model & $\alpha$ & $K_{1}(\mathrm{mJy})$ & $K_{2} \times 10^{4}$ & $v_{\mathrm{c}, 2}(\mathrm{MHz})$ & $K_{3} \times 10^{4}$ & $v_{\mathrm{c}, 3}(\mathrm{MHz})$ & $\chi_{\mathrm{red}}^{2}$ \\
\hline 1 & $-0.65 \pm 0.02$ & $14.4 \pm 0.4$ & - & - & - & - & 8.9 \\
2 & $-0.67 \pm 0.02$ & $14.4 \pm 0.2$ & $8 \pm 3$ & $169_{-38}^{+31}$ & - & - & 3.6 \\
3 & $-0.67 \pm 0.02$ & $14.4 \pm 0.2$ & - & - & $17 \pm 7$ & $241_{-52}^{+42}$ & 3.6 \\
\hline
\end{tabular}

$\alpha$ is the observed spectral index; $K_{1}$ is the flux density at a frequency of $5 \mathrm{GHz} ; K_{2}$ and $K_{3}$ are the optical depths at $5 \mathrm{GHz}$ of the homogeneous and "clumpy" external absorbing media, respectively; $\chi_{\text {red }}^{2}$ is the $\chi^{2}$ per degree of freedom. Models 1 through 3 are explained in the text. $\left(v_{\mathrm{c}, 2}\right.$ and $v_{\mathrm{c}, 3}$ are not fitted, and correspond to the frequencies at which the free-free optical depth is unity for the homogeneous and "clumpy" medium, respectively.)

predict that, for the physical parameters they are considering, radiative (synchrotron) losses start to become less important at $t \geq 1000$ days at frequencies below $\sim 2.3 \mathrm{GHz}$, whereas energy losses of the electrons due to adiabatic and Coulomb cooling contribute significantly below $2.3 \mathrm{GHz}$, which seems consistent with the observational results. We note that a multifrequency monitoring of SN 1993J at radio frequencies down to $\sim 70 \mathrm{MHz}$, would likely prove to be very useful to further constrain the population of electrons responsible for the emission, as well as to better understand the late-time radio evolution of SN 1993J.

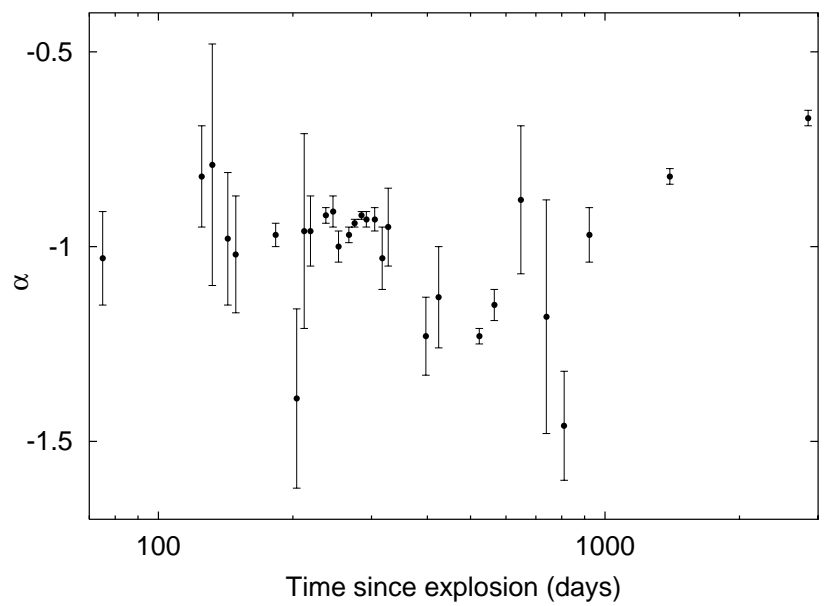

Fig. 3. Evolution of the observed spectral index, $\alpha$, for SN 1993J, as obtained from fitting the radio continuum data at each epoch to model 2 (see text for details).

Figure 4 shows the fits of model 2 to all data points (solid line), and to all but the $P$-band data point (dashed line). Because any absorption model will obviously fit by turning down sharply below the lowest frequency data point, the availability of radio continuum measurements at the lowest possible frequencies is important, in particular as a radio supernova ages. Had the $P$-band data not been available, we could have wrongly concluded that the peak of the SN 1993J spectrum is at $v \sim 0.8 \mathrm{GHz}$ at a level of $\sim 35 \mathrm{mJy}$. Having the $P$-data point available, we conclude that the peak is around, or below $0.3 \mathrm{GHz}$, and at least at a level of $70 \mathrm{mJy}$. (We note that a similar fit to all data, but using model 3 rather than model 2, would not change this result.) Similarly, the best fit to our SN 1993J radio data using models 2 or 3 as described above, but not

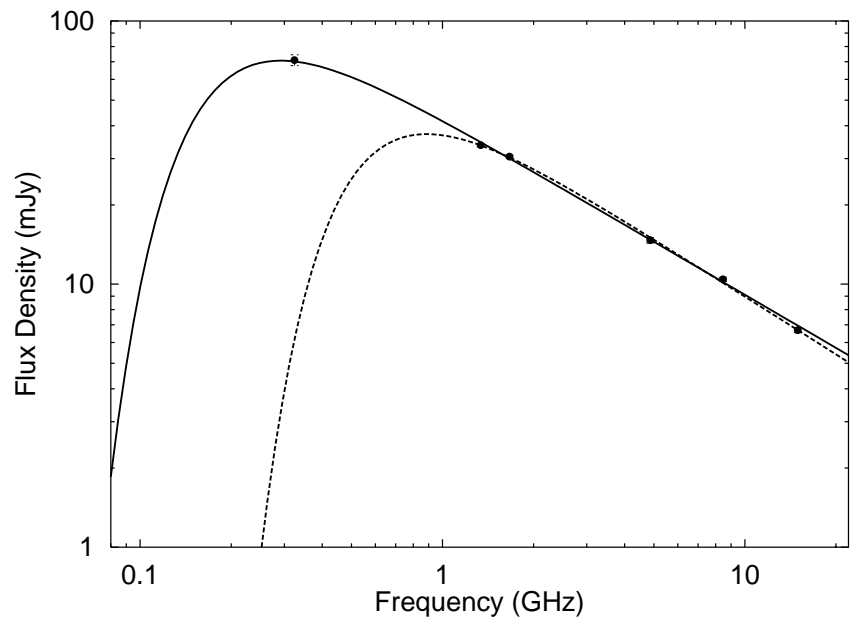

Fig. 4. Fits to the continuum radio spectrum of SN 1993J on 17 and 21 December 2000. Although both fits correspond to model 2, in one case (solid line) all radio measurements are used, while in the other case (dashed line) all but the $P$-band data point are used. This results in a turnover frequency much higher than actually observed. See text for details.

including the $P$-band measurement, yields $\alpha=-0.73$, a somewhat steeper value than is obtained if all data points are used, $\alpha=-0.67$, but within $2 \sigma$ of it.

\section{Summary}

We present VLA radio continuum measurements of SN 1993J in M 81 taken on 17 and 21 December, 2000, about 2820 days after the supernova explosion, spanning the frequency range from 0.32 to $14.9 \mathrm{GHz}$. We discuss in detail the $P$-band (322$327 \mathrm{MHz}$ ) observations, since the task of obtaining the flux density at these frequencies is much harder than at higher frequencies, due to confusion from many nearby and far away, strong sources, e.g. M 82. We also point out the importance of having a radio spectral coverage that extends down to $P$-band as the supernova ages, since otherwise the conclusions can be wrong.

We discuss three models for the late radio emission of SN 1993J: (i) a simple power-law spectrum, (ii) a power-law spectrum free-free absorbed by a screen of homogeneously distributed ionized gas, and (iii) a power-law spectrum free-free absorbed by a "clumpy" medium. We find that the best fit to the data is yielded by a power-law spectrum free-free absorbed by 
either a homogeneous, or a clumpy, distribution of ionized gas. However, a mixture of homogeneous and clumpy absorbers, while not preferred, cannot be totally ruled out. The radio spectrum between 0.32 and $14.94 \mathrm{GHz}$ is well characterized by $\alpha=-0.67 \pm 0.02\left(S_{v} \propto v^{\alpha}\right)$, typical of an optically thin radio supernova, and is significantly different from that obtained at epochs $\lesssim 1000$ days between 1.4 and $14.9 \mathrm{GHz}$, which indicates an ongoing spectral evolution in the radio emission from SN 1993J. Since the spectral index $\gamma$ of the electron distribution $\left(N_{E}=N_{0} E^{-\gamma}\right)$, is related to the observed spectral index by $\gamma=1-2 \alpha$, our value of $\alpha$ translates into $\gamma=2.34 \pm 0.04$, which agrees well with that predicted by Fransson $\&$ Björnsson (1998) for $t \gtrsim 1000$ days. We also fit the available radio continuum data of SN 1993J for the period from 70 up to 2820 days since the explosion, using a power-law spectrum free-free absorbed by a screen of homogeneously distributed ionized gas. The fit shows that the observed spectral index of SN 1993J has been slowly evolving since $t \sim 1000$ days on, with $\alpha$ increasing from a value close to -1 to the current -0.67 . This spectral evolution seems to suggest that, in addition to the radiative (synchrotron) losses, adiabatic cooling and ionization (Coulomb) losses at the lowest frequencies might be contributing significantly to the integrated electron spectrum. An extension of the low frequency observations further down to $\sim 70 \mathrm{MHz}$ should help to fine tune the physical parameters of SN 1993J, and discern between the homogeneous or clumpy nature of its circumstellar medium. From a technical point of view, such observations will be challenging.

Acknowledgements. We thank Lucas Lara for his help with some aspects of the data reduction process, and Gianfranco Brunetti for discussions. We thank Kurt Weiler for making the VLA data publicly available. We are also grateful to an anonymous referee for a careful and constructive review of our manuscript. MAPT is grateful to the Instituto de Astrofísica de Andalucía for its hospitality during a short visit. This research has been supported by a Marie Curie Fellowship of the European Community (contract IHPMCFI-99-1), and by the Spanish DGICYT grants AYA2001-2147C02-01 and AYA2001-2147-C02-02. The VLA is an instrument of the National Radio Astronomy Observatory, which is a facility of the National Science Foundation operated under cooperative agreement by Associated Universities, Incorporated.

\section{References}

Bartel, N., Bietenholz, M. F., Rupen, M. P., et al. 2000, Science, 287, 112

Chevalier, R. A. 1982a, ApJ, 259, 302

Chevalier, R. A. 1998, ApJ, 499, 810

Fransson, C., \& Björnsson, C.-I. 1998, ApJ, 509, 861

Immler, S., Aschenbach, B., \& Wang, Q. D. 2001, ApJ, 561, L107

Lundqvist, P. 1994, in Circumstellar Media in the Late Stages of Stellar Evolution, ed. R. E. S. Clegg, I. R. Stevens, \& W. P. S. Meikle (Cambridge: Cambridge University Press), 213

Marcaide, J. M., Alberdi, A., Ros, E., et al. 1995a, Nature, 373, 44

Marcaide, J. M., Alberdi, A., Ros, E., et al. 1995b, Science, 270, 1475

Marcaide, J. M., Alberdi, A., Ros, E., et al. 1997, ApJ, 486, L31

Mioduszewski, A. J., Dwarkadas, V. V., \& Ball, L. 2001, ApJ, 562, 869

Pérez-Torres, M. A., Alberdi, A., \& Marcaide, J. M. 2001, A\&A, 374, 997

Van Dyk, S. D., Weiler, K. W., Sramek, R. A., et al. 1994, ApJ, 432, L115

Weiler, K. W., Sramek, R. A., Panagia, N., et al. 1986, ApJ, 301, 790

Weiler, K. W., Panagia, N., Sramek, R. A., et al. 1989, ApJ, 336, 421

Weiler, K. W., Panagia, N., \& Sramek, R. A. 1990, ApJ, 364, 611 